\title{
Coffee consumption and risk of total and cardiovascular mortality among patients with type 2 diabetes
}

\author{
S. Bidel • G. Hu • Q. Qiao • P. Jousilahti • R. Antikainen • \\ J. Tuomilehto
}

Received: 14 March 2006 / Accepted: 27 July 2006 / Published online: 21 September 2006

(C) Springer-Verlag 2006

\begin{abstract}
Aims/hypothesis Higher habitual coffee drinking has been associated with a lower risk of developing type 2 diabetes. The relation between coffee consumption and risk of cardiovascular disease (CVD) has been examined in many studies, but the issue remains controversial. This study was designed to assess the association between coffee consumption and CVD mortality among patients with type 2 diabetes.

Methods We prospectively followed 3,837 randomly ascertained Finnish patients with type 2 diabetes aged 25 to
\end{abstract}

S. Bidel and G. Hu have contributed equally to this manuscript as first authors.

S. Bidel $(\bowtie) \cdot \mathrm{G} . \mathrm{Hu} \cdot \mathrm{Q}$. Qiao $\cdot$ P. Jousilahti $\cdot$ J. Tuomilehto

Diabetes and Genetic Epidemiology Unit,

Department of Health Promotion and Chronic Disease Prevention,

National Public Health Institute,

Mannerheimintie 166,

FIN-00300 Helsinki, Finland

e-mail: siamak.bidel@ktl.fi

S. Bidel $\cdot$ G. Hu $\cdot$ Q. Qiao $\cdot$ J. Tuomilehto

Department of Public Health, University of Helsinki,

Helsinki, Finland

P. Jousilahti

School of Public Health, University of Tampere,

Tampere, Finland

R. Antikainen

Oulu City Hospital and Department of Internal Medicine,

University of Oulu,

Oulu, Finland

J. Tuomilehto

South Ostrobothnia Central Hospital,

Seinäjoki, Finland
74 years. Coffee consumption and other study parameters were determined at baseline. The International Classification of Diseases was used to identify CHD, CVD and stroke cases using computerised record linkage to the national Death Registry. The associations between coffee consumption at baseline and risk of total, CVD, CHD, and stroke mortality were analysed by using Cox proportional hazards models.

Results During the average follow-up of 20.8 years, 1,471 deaths were recorded, of which 909 were coded as CVD, 598 as CHD and 210 as stroke. The respective multivariateadjusted hazard ratios in participants who drank 0-2, 3-4, $5-6$, and $\geq 7$ cups of coffee daily were $1.00,0.77,0.68$ and 0.70 for total mortality $(P<0.001$ for trend), $1.00,0.79,0.70$ and 0.71 for CVD mortality ( $P=0.006$ for trend), $1.00,0.78$, 0.70 and 0.63 for CHD mortality ( $p=0.01$ for trend), and $1.00,0.77,0.64$ and 0.90 for stroke mortality $(p=0.12$ for trend).

Conclusions/interpretation In this large prospective study we found that in type 2 diabetic patients coffee drinking is associated with reduced total, CVD and CHD mortality.

Keywords Cardiovascular disease - Coffee consumption . Stroke $\cdot$ Total mortality Type 2 diabetes

\section{Abbreviations \\ CVD cardiovascular disease \\ HRs hazard ratios}

\section{Introduction}

Type 2 diabetes is characterised by the progressive development of microvascular and macrovascular compli- 
cations. These complications, and in particular atherosclerotic lesions, are the main causes for most of the increased morbidity and mortality in type 2 diabetes and persons with the disease are at much higher risk of fatal ischaemic heart disease and stroke [1-3]. In general type 2 diabetes increases the risk of cardiovascular disease (CVD) mortality by about threefold compared with non-diabetic subjects. Suggested mechanisms for this effect include increased atherosclerosis, hypercoagulability and hypertension, but a complete explanation has yet to be formulated [4].

Coffee is probably one of the most frequently consumed beverages in the world, and recent cohort studies [5-7] and a systematic review by Van Dam et al. [8] revealed that coffee consumption has been associated with a substantially lower risk of developing type 2 diabetes. On the other hand, in the many studies examining the role of coffee consumption in the risk of CVD, most [9-12], but not all [13-16], including those that found coffee to be a CVD risk augmenter, found no association. In a newly published investigation [17], the authors found that coffee may increase risk of non-fatal myocardial infarction, but only among individuals with a certain genotype and with slow caffeine metabolism. Moreover, no previous studies have examined the association between coffee consumption and the risk of CVD mortality among patients with type 2 diabetes. The aim of this study therefore was to examine the association between coffee consumption and the risk of total, CVD, CHD and stroke mortality among Finnish type 2 diabetic patients participating in population-based surveys.

\section{Subjects and methods}

\section{Participants}

Six independent cross-sectional population surveys were carried out in the Kuopio and North Karelia provinces in eastern Finland in 1972, 1977, 1982, 1987, 1992 and 1997 [18]. The survey was expanded to the Turku-Loimaa region in southwestern Finland in 1982, to the Helsinki capital area in 1992 and to the northern province of Oulu in 1997. In 1972 and 1977, a randomly selected sample (6.6\%) of the population born between 1913 and 1947 was drawn. Since 1982, the sample has been stratified by area, sex and 10-year age group according to the WHO 'Monitoring trends and determinants of cardiovascular disease' protocol [19]. In the six surveys, the subjects included were 25 to 64 years of age, and in the 1997 survey subjects between the ages of 65 and 74 years were also included. Subjects who participated in more than one survey were included only in the first survey cohort. The total sample size of the six surveys was 53,166 . The participation rate varied by year from $74 \%$ to $88 \%$ [18]. All participants gave informed consent (verbal for the years 1972-1992, written for 1997). These surveys were conducted according to the ethical rules of the National Public Health Institute, and the investigations were carried out in accordance with the Declaration of Helsinki.

Among the subjects who participated in the abovementioned six independent cross-sectional population surveys, diabetes, and therefore eligibility for inclusion in the present analysis, was established according to the following criteria: (1) subject reported having diabetes on the questionnaire at the baseline survey; (2) subject had a hospital discharge diagnosis of diabetes between 1968 and 2002; (3) subject was entitled to special reimbursement for anti-diabetic drugs according to the National Social Insurance Institution's Drug Register between 1964 and 2002. The National Hospital Discharge Register and Drug Register data were linked to the risk factor survey data with the unique personal identification numbers assigned to every resident of Finland. The Hospital Discharge Register has reported the codes for type 1 and type 2 diabetes separately since 1987 . Glucose-lowering drugs prescribed by a physician are free of charge in Finland and subject to approval of the Social Insurance Institution based on a review of each case history. The physician confirms the diagnosis of diabetes by applying the WHO criteria, i.e.: (1) one or more classic symptoms plus a fasting plasma glucose level $\geq 7.8 \mathrm{mmol} / \mathrm{l}(\geq 7.0 \mathrm{mmol} / \mathrm{l}$ from 1998) or an OGTT result $\geq 11.1 \mathrm{mmol} / \mathrm{l}$; (2) at least one raised plasma glucose concentration for fasting plasma glucose, i.e. $\geq 7.8 \mathrm{mmol} / 1$ ( $\geq 7.0 \mathrm{mmol} / \mathrm{l}$ from 1998) or for the OGTT, i.e. $\geq 11.1 \mathrm{mmol} / 1$ in the absence of symptoms; or (3) treatment with a hypoglycaemic drug (oral antidiabetic agents or insulin) [20, 21] All approvals of patients receiving free medication are entered into a register maintained by the Social Insurance Institution. A total of 4,453 people were recognised as having diabetes. Of these, 503 were excluded because they had either CHD or stroke at baseline or had type 1 diabetes at baseline or detected during follow-up; another 113 were excluded because data on coffee consumption, physical activity, BMI, blood pressure, serum cholesterol level or smoking status were incomplete. Thus, 3,837 patients were included in the present study.

\section{Measurements}

A self-administered questionnaire was sent to the participants to be completed at home. The questionnaire included questions on medical history, socioeconomic factors, physical activity, coffee, tea and alcohol consumption, and smoking habits. Education level, measured as the total 
number of school years, was divided into birth-cohortspecific tertiles. Based on the responses, the participants were classified as never, ex- and current-smokers. Current smokers were categorised into those who smoked $<20$, or $\geq 20$ cigarettes per day. Physical activity included occupational, commuting and leisure time physical activity. The subjects reported their occupational physical activity according to the following three categories: low, moderate, and high. Self-reported leisure time physical activity was classified into three categories: low, moderate, and high. The daily commuting return journey was grouped into three categories: (1) those using motorised transportation or not in work (0 min of walking or cycling); (2) those walking or bicycling 1 to $29 \mathrm{~min}$; (3) those walking or bicycling for more than $30 \mathrm{~min}$. Since our group has found that moderate and high occupational, commuting ( $\geq 1 \mathrm{~min}$ ) or leisure-time physical activity independently and significantly reduces risk of total and CVD mortality among diabetic patients $[22,23]$, physical activities were merged and regrouped into three categories: (1) low for subjects who reported light levels of occupational, commuting $(<1 \mathrm{~min})$ and leisuretime physical activity; (2) moderate for subjects who reported moderate to high physical activity in only one of the three categories; (3) high for subjects who reported moderate to high physical activity in two or three of the three categories.

The participants were asked: 'How many cups of coffee or tea do you drink per day (one cup of coffee equal to $100 \mathrm{ml}$; one cup of tea equal to $200 \mathrm{ml}$ )?' [11] Coffee consumption was categorised into five categories: 0-2 cups, 3-4 cups, 5-6 cups, and $\geq 7$ cups. As only a few people drank tea, tea consumption was categorised into three categories: none, $1-2$ cups, and $\geq 3$ cups. Since questions on alcohol consumption were worded differently between the first two surveys (1972 and 1977) and the latter surveys, participants were categorised into abstainers and alcohol users.

At the study site, specially trained research nurses checked that the questionnaire was fully completed and measured blood pressure, height and weight using a standardised protocol [20]. Blood pressure was measured after 5 min of rest using a standard mercury manometer. Height was measured without shoes. Weight was measured with light clothing. BMI was calculated as weight in kilograms divided by the square of the height in metres $\left(\mathrm{kg} / \mathrm{m}^{2}\right)$. After blood pressure measurement, a venous blood specimen was drawn. Serum total cholesterol was determined using the Lieberman-Burchard method in 1972 and 1977, and an enzymatic method (CHOD-PAP; Boehringer Mannheim, Mannheim, Germany) from 1982. The enzymatic assay method gave values $2.4 \%$ lower than the Lieberman-Burchard method. The cholesterol values from 1972 and 1977 were corrected by this percentage. All samples were analysed in the same laboratory at the National Public Health Institute.

Prospective follow-up

The study cohorts were followed until the end of 2003 through computerised register linkage. Mortality data were obtained from Statistics Finland and were linked with the survey data using the personal identification number assigned to every resident in Finland. The International Classification of Diseases (8th, 9th and 10th Revisions) were used to identify deaths from CHD (410-414 and I20I25), stroke (430-438 and I60-I66), and CVD (390-459 and I00-I99).

\section{Statistical analysis}

Differences in risk factors at different levels of coffee consumption were tested using analysis of variance or logistic regression after adjustment for age, sex, and study year. The associations between coffee consumption at baseline and the risk of total, CVD, CHD and stroke mortality were analysed by using Cox proportional hazards models. Different levels of coffee consumption were included in the models as dummy variables, and the significance of the trend over different categories of coffee consumption was tested in the same models by assigning an ordinal numeric value to each dummy variable. The proportional hazards assumption in the Cox model was assessed with graphical methods and with models including time-by-covariate interactions [24]. In general, all proportionality assumptions were appropriate. All analyses were adjusted for age, study year, BMI, systolic blood pressure, total cholesterol, education, physical activity, alcohol and tea consumption, and smoking. The reference category of 0-2 cups per day was chosen, rather than the non-coffee drinking category, for four reasons: (1) the risk of mortality did not differ between coffee drinkers with 1-2 cups daily and coffee abstainers (hazard ratios [HRs]: 1.04 for total mortality and 0.98 for CVD mortality); (2) the risk of mortality decreased in coffee drinkers with 3-4 cups daily compared with coffee abstainers (HRs: 0.81 for total mortality and 0.77 for CVD mortality); (3) the risk of mortality decreased significantly in coffee drinkers with 34 cups daily compared with coffee drinkers with 1-2 cups daily (HRs: 0.74 for total mortality and 0.75 for CVD mortality); (4) the 0-2 cups reference group contained larger numbers of participants. Since the interactions of sex and coffee consumption on mortality were not statistically significant, men and women were combined in the analyses. Statistical significance was considered to be $p<0.05$. All statistical analyses were performed using SPSS for Windows 13.0 (SPSS, Chicago, IL, USA). 


\section{Results}

During the average follow-up of 20.8 years, 1,471 deaths were recorded, of which 909 were coded as CVD, 598 as CHD and 210 as stroke. The baseline characteristics of the study population are presented in Table 1 . The median coffee consumption daily was 5.0 cups (interquartile range 3.0-6.0 cups). After adjustment for age, sex and study year, coffee consumption had a direct association with total cholesterol level and cigarette smoking, and an inverse association with education level, low physical activity and tea drinking.

The respective HRs, adjusted for age, sex and study year, in participants who drank $0-2,3-4,5-6$, and $\geq 7$ cups of coffee daily were $1.00,0.79,0.72,0.79$ for total mortality ( $p=0.001$ for trend), 1.00, 0.81, 0.75, 0.79 for CVD mortality $(P=0.04$ for trend), $1.00,0.81,0.77,0.71$ for CHD mortality ( $P=0.09$ for trend), and $1.00,0.75,0.62$, 0.90 for stroke mortality ( $p=0.08$ for trend) (Table 2). After further adjustment for BMI, systolic blood pressure, total cholesterol, education, physical activity, alcohol and tea consumption, and smoking, these inverse associations became stronger and were significant for total mortality ( $p<0.001$ for trend), CVD mortality ( $p=0.006$ for trend) and CHD mortality ( $p=0.01$ for trend), but no longer significant for stroke mortality ( $p=0.12$ for trend). When we included all three types of physical activity (occupational, commuting, leisure time) in the analysis, these associations did not appreciably change (results not shown).

When coffee consumption was examined as a continuous variable, the respective multivariate-adjusted HRs associated with an increment of one cup of coffee per day were 0.96 (95\% CI $0.94-0.99 ; p=0.002)$ for total mortality, 0.97 (95\% CI $0.94-0.99 ; p=0.02$ ) for CVD mortality, 0.96 (95\% CI $0.93-0.99 ; p=0.02$ ) for CHD mortality, and 0.99 (95\% CI $0.92-1.05 ; p=0.72$ ) for stroke mortality (Table 3 ).
We also compared mortality in people drinking 0-2 cups of coffee with that in people drinking 3 cups of coffee or more. The multivariate-adjusted HRs in people drinking 3 cups of coffee or more compared with people drinking $0-2$ cups of coffee daily were 0.72 (95\% CI $0.62-0.83$; $p<$ $0.001)$ for total mortality, 0.73 (95\% CI 0.61-0.89; $p=$ 0.001 ) for CVD mortality, 0.71 (95\% CI 0.57-0.90; $p=0.005$ ) for CHD mortality, and 0.73 (95\% CI 0.50 $1.09 ; p=0.12$ ) for stroke mortality (Table 3 ).

There was a non-significant inverse association between tea consumption and mortality from all causes, CVD and CHD (Table 4). When tea drinkers were combined as one group, the multivariate-adjusted HRs in tea drinkers versus tea abstainers were $0.85(95 \%$ CI $0.75-0.96 ; p=0.008)$ for total mortality, $0.85(95 \%$ CI $0.73-0.99 ; p=0.04)$ for CVD mortality, and 0.83 (95\% CI $0.69-1.00 ; p=0.05)$ for $\mathrm{CHD}$ mortality.

A multivariate adjusted inverse association between coffee consumption and total mortality was present in subjects aged 25 to 54 years ( $p<0.001$ for trend) and 55 to 74 years ( $p=0.06$ for trend), both in men ( $p=0.004$ for trend) and in women ( $p=0.02$ for trend) (Table 5). The multivariate-adjusted HRs for total mortality in people drinking 3 cups of coffee or more compared with people drinking $0-2$ cups of coffee daily were 0.67 (95\% CI $0.54-0.82$; $p<0.001)$ in subjects aged 25 to 54 years, $0.76(95 \% \mathrm{CI}$, $0.61-0.94 ; p=0.01)$ in subjects aged 55 to 74 years, 0.71 $(95 \%$ CI $0.57-0.87 ; p=0.001)$ in men, and $0.73(95 \%$ CI $0.59-0.90 ; p=0.004)$ in women. A statistically significant inverse association between coffee consumption and CVD mortality was found in subjects aged 25 to 54 years $(p=0.01$ for trend), and in women ( $p=0.03$ for trend). The inverse association between coffee consumption and CHD mortality was statistically significant in subjects aged 25 to 54 years $(p=0.04$ for trend).
Table 1 Baseline characteristics by volume of coffee consumption among subjects with type 2 diabetes

Values represent means $\pm \mathrm{SD}$ or percentages unless otherwise indicated. Adjusted for age, sex, and study year

${ }^{a}$ One cup of coffee $=100 \mathrm{ml}$

\begin{tabular}{|c|c|c|c|c|c|}
\hline & \multicolumn{4}{|c|}{ Daily coffee consumption (no. of cups) ${ }^{a}$} & \multirow[t]{2}{*}{$p$ value } \\
\hline & $0-2$ & $3-4$ & $5-6$ & $\geq 7$ & \\
\hline Number of participants & 644 & 1,041 & 1,356 & 796 & \\
\hline Women $(\%)$ & 47.2 & 58.6 & 54.6 & 38.3 & \\
\hline Age, years & $48.8 \pm 11.3$ & $49.7 \pm 10.3$ & $49.4 \pm 10.3$ & $47.8 \pm 9.9$ & $<0.001$ \\
\hline BMI $\left(\mathrm{kg} / \mathrm{m}^{2}\right)$ & $29.4 \pm 5.1$ & $29.8 \pm 5.1$ & $30.0 \pm 5.2$ & $29.9 \pm 5.2$ & 0.07 \\
\hline Systolic blood pressure (mmHg) & $152 \pm 23$ & $153 \pm 24$ & $153 \pm 24$ & $153 \pm 24$ & $>0.2$ \\
\hline Diastolic blood pressure $(\mathrm{mmHg})$ & $93 \pm 13$ & $93 \pm 13$ & $93 \pm 13$ & $92 \pm 13$ & $>0.2$ \\
\hline Total cholesterol (mmol/l) & $6.3 \pm 1.3$ & $6.5 \pm 1.3$ & $6.6 \pm 1.4$ & $6.6 \pm 1.3$ & 0.001 \\
\hline Education (years) & $8.3 \pm 3.9$ & $8.0 \pm 3.6$ & $7.5 \pm 3.2$ & $7.2 \pm 3.1$ & $<0.001$ \\
\hline Low physical activity (\%) & 22.7 & 18.4 & 15.6 & 13.1 & $<0.001$ \\
\hline Alcohol drinkers $(\%)$ & 43.0 & 38.1 & 38.4 & 44.0 & 0.2 \\
\hline Tea drinkers (\%) & 58.1 & 39.9 & 21.3 & 11.4 & $<0.001$ \\
\hline Current smoking (\%) & 22.8 & 20.7 & 25.5 & 45.9 & $<0.001$ \\
\hline
\end{tabular}


Table 2 HRs (95\% CIs) of total, CVD, CHD and stroke mortality by volume of coffee consumption among subjects with type 2 diabetes

\begin{tabular}{|c|c|c|c|c|c|}
\hline & \multicolumn{4}{|c|}{ Daily coffee consumption (no. of cups) ${ }^{\mathrm{a}}$} & \multirow[t]{2}{*}{$p$ value for trend } \\
\hline & $0-2$ & $3-4$ & $5-6$ & $\geq 7$ & \\
\hline \multicolumn{6}{|l|}{ Total mortality } \\
\hline Number of deaths & 247 & 384 & 529 & 311 & \\
\hline Person-years & 11,772 & 20,551 & 29,927 & 17,406 & \\
\hline HRs, model $1^{\mathrm{b}}$ & 1.00 & $0.79(0.67-0.93)$ & $0.72(0.62-0.85)$ & $0.79(0.67-0.94)$ & 0.001 \\
\hline HRs, model $1^{\mathrm{c}}$ & 1.00 & $0.78(0.66-0.92)$ & $0.69(0.59-0.82)$ & $0.72(0.60-0.87)$ & $<0.001$ \\
\hline HRs, model $1^{\mathrm{d}}$ & 1.00 & $0.77(0.65-0.91)$ & $0.68(0.58-0.80)$ & $0.70(0.59-0.85)$ & $<0.001$ \\
\hline \multicolumn{6}{|l|}{ CVD mortality } \\
\hline Number of deaths & 146 & 241 & 337 & 185 & \\
\hline HRs, model $1^{\mathrm{b}}$ & 1.00 & $0.81(0.66-0.99)$ & $0.75(0.62-0.92)$ & $0.79(0.63-0.98)$ & 0.04 \\
\hline HRs, model $1^{\mathrm{c}}$ & 1.00 & $0.80(0.65-0.99)$ & $0.73(0.59-0.90)$ & $0.74(0.58-0.94)$ & 0.02 \\
\hline HRs, model $1^{\mathrm{d}}$ & 1.00 & $0.79(0.64-0.97)$ & $0.70(0.57-0.86)$ & $0.71(0.56-0.90)$ & 0.006 \\
\hline \multicolumn{6}{|l|}{ CHD mortality } \\
\hline Number of deaths & 96 & 160 & 231 & 111 & \\
\hline HRs, model $1^{\mathrm{b}}$ & 1.00 & $0.81(0.63-1.05)$ & $0.77(0.61-0.98)$ & $0.71(0.54-0.94)$ & 0.09 \\
\hline HRs, model $1^{\mathrm{c}}$ & 1.00 & $0.79(0.61-1.03)$ & $0.73(0.57-0.95)$ & $0.65(0.48-0.88)$ & 0.04 \\
\hline HRs, model $1^{\mathrm{d}}$ & 1.00 & $0.78(0.60-1.01)$ & $0.70(0.54-0.90)$ & $0.63(0.47-0.84)$ & 0.01 \\
\hline \multicolumn{6}{|l|}{ Stroke mortality } \\
\hline Number of deaths & 35 & 54 & 69 & 52 & \\
\hline HRs, model $1^{\mathrm{b}}$ & 1.00 & $0.75(0.49-1.14)$ & $0.62(0.41-0.94)$ & $0.90(0.59-1.40)$ & 0.08 \\
\hline HRs, model $1^{\mathrm{c}}$ & 1.00 & $0.79(0.51-1.22)$ & $0.66(0.43-1.03)$ & $0.94(0.58-1.52)$ & 0.15 \\
\hline HRs, model $1^{\mathrm{d}}$ & 1.00 & $0.77(0.50-1.19)$ & $0.64(0.41-0.99)$ & $0.90(0.56-1.45)$ & 0.12 \\
\hline
\end{tabular}

\section{Discussion}

In this large prospective study we found an inverse association between coffee consumption and the risk of total, CVD and CHD mortality among patients with type 2 diabetes. Daily tea drinking was modestly and inversely associated with the risk of total, CVD and CHD mortality among patients with type 2 diabetes. These associations were independent of age, BMI, systolic blood pressure, total cholesterol, physical activity, alcohol drinking and smoking.
Types 2 diabetes is a well-known risk factor for allcause, CVD and stroke mortality [23, 25-28]. A doseresponse relationship between level of hyperglycaemia and both CVD deaths and all-cause mortality has been observed [27]. Recently clear evidences released from cohort studies [5-7] and a systematic review [8] revealed that the risk of type 2 diabetes among habitual coffee consumers is substantially lower than among non-coffee drinkers. On the other hand, the relation between coffee consumption and development or progression of CVD remains controversial. Most of the previous cohort studies found no

Table 3 HRs (95\% CIs) of total, CVD, CHD and stroke mortality by volume of coffee as continuous variable and by different volume of coffee ( $\geq 3$ cups vs $0-2$ cups) among subjects with type 2 diabetes

\begin{tabular}{|c|c|c|c|c|}
\hline & \multicolumn{2}{|c|}{ Coffee as continuous variable } & \multicolumn{2}{|c|}{$\geq 3$ cups vs $0-2$ cups of coffee daily ${ }^{a}$} \\
\hline & HRs (95\% CIs) & $p$ value & HRs (95\% CIs) & $p$ value \\
\hline Total mortality & $0.96(0.94-0.99)$ & 0.002 & $0.72(0.62-0.83)$ & $<0.001$ \\
\hline Cardiovascular mortality & $0.97(0.94-0.99)$ & 0.02 & $0.73(0.61-0.89)$ & 0.001 \\
\hline Coronary heart disease mortality & $0.96(0.93-0.99)$ & 0.02 & $0.71(0.57-0.90)$ & 0.005 \\
\hline Stroke mortality & $0.99(0.92-1.05)$ & $>0.2$ & $0.73(0.92-1.05)$ & 0.12 \\
\hline
\end{tabular}

Values adjusted for age, sex, study year, BMI, systolic blood pressure, total cholesterol, education, alcohol and tea consumption, and smoking status

${ }^{\text {a }}$ One cup of coffee $=100 \mathrm{ml}$ 
Table 4 HRs (95\% CIs) of total, CVD, CHD and stroke mortality by volume of tea consumption among subjects with type 2 diabetes

${ }^{\text {a }}$ One cup of tea $=200 \mathrm{ml}$

${ }^{\mathrm{b}}$ Adjusted for age, sex, and study year

${ }^{c}$ Adjusted for age, sex, study year, education, alcohol and tea consumption, and smoking status

${ }^{\mathrm{d}}$ Adjusted for age, sex, study year, BMI, systolic blood pressure, total cholesterol, education, alcohol and coffee consumption, and smoking status

\begin{tabular}{|c|c|c|c|c|}
\hline & \multicolumn{3}{|c|}{ Daily tea consumption, cups $^{\mathrm{a}}$} & \multirow[t]{2}{*}{$p$ value for trenc } \\
\hline & 0 & $1-2$ & $\geq 3$ & \\
\hline \multicolumn{5}{|l|}{ Total mortality } \\
\hline Number of deaths & 1,056 & 296 & 119 & \\
\hline Person-years & 55,944 & 17,436 & 6,276 & \\
\hline HRs, model $1^{\mathrm{b}}$ & 1.00 & $0.88(0.77-1.00)$ & $0.92(0.76-1.11)$ & 0.13 \\
\hline HRs, model $1^{\mathrm{c}}$ & 1.00 & $0.84(0.74-0.97)$ & $0.85(0.70-1.04)$ & 0.03 \\
\hline HRs, model $1^{\mathrm{d}}$ & 1.00 & $0.84(0.73-0.96)$ & $0.87(0.71-1.06)$ & 0.03 \\
\hline \multicolumn{5}{|l|}{ CVD mortality } \\
\hline Number of deaths & 652 & 186 & 71 & \\
\hline HRs, model $1^{\mathrm{b}}$ & 1.00 & $0.89(0.76-1.05)$ & $0.88(0.69-1.12)$ & $>0.2$ \\
\hline HRs, model $1^{\mathrm{c}}$ & 1.00 & $0.86(0.72-1.02)$ & $0.82(0.63-1.06)$ & 0.10 \\
\hline HRs, model $1^{\mathrm{d}}$ & 1.00 & $0.85(0.71-1.00)$ & $0.85(0.66-1.10)$ & 0.11 \\
\hline \multicolumn{5}{|l|}{ CHD mortality } \\
\hline Number of deaths & 430 & 126 & 42 & \\
\hline HRs, model $1^{\mathrm{b}}$ & 1.00 & $0.92(0.75-1.12)$ & $0.78(0.57-1.07)$ & $>0.2$ \\
\hline HRs, model $1^{\mathrm{c}}$ & 1.00 & $0.87(0.70-1.07)$ & $0.72(0.52-1.00)$ & 0.09 \\
\hline HRs, model $1^{\mathrm{c}}$ & 1.00 & $0.85(0.69-1.05)$ & $0.76(0.54-1.05)$ & 0.13 \\
\hline \multicolumn{5}{|l|}{ Stroke mortality } \\
\hline Number of deaths & 143 & 44 & 23 & \\
\hline HRs, model $1^{\mathrm{a}}$ & 1.00 & $0.97(0.69-1.37)$ & $1.28(0.82-1.98)$ & $>0.2$ \\
\hline HRs, model $1^{\mathrm{c}}$ & 1.00 & $0.97(0.68-1.39)$ & $1.23(0.77-1.95)$ & $>0.2$ \\
\hline HRs, model $1^{\mathrm{d}}$ & 1.00 & $0.96(0.67-1.37)$ & $1.26(0.79-2.00)$ & $>0.2$ \\
\hline
\end{tabular}

evidence of any positive association between coffee consumption and risk of CHD [9-12], while a Scottish cohort study [13] revealed that higher coffee consumption was associated with lower risk of CVD among men but not women. Kleemola et al. [14] in a study of a Finnish cohort observed a lower risk of non-fatal myocardial infarction in men with higher coffee consumption ( $>7$ cups per day). They also found a slight increase in CHD mortality in the
Table 5 HRs (95\% CIs) of total, CVD and CHD mortality by volume of coffee consumption among subjects with type 2 diabetes by various subpopulations
Values adjusted for age, sex (excluding in sex analysis), study year, BMI, systolic blood pressure, total cholesterol, education, alcohol and tea consumption, and smoking status ${ }^{\text {a }}$ One cup of coffee $=100 \mathrm{ml}$

\begin{tabular}{|c|c|c|c|c|c|}
\hline \multirow[t]{2}{*}{ HRs (95\% CIs) } & \multicolumn{4}{|c|}{ Daily coffee consumption, cups $^{\mathrm{a}}$} & \multirow[t]{2}{*}{$p$ value for trend } \\
\hline & $0-2$ & $3-4$ & $5-6$ & $\geq 7$ & \\
\hline \multicolumn{6}{|l|}{ Total mortality } \\
\hline \multicolumn{6}{|l|}{ Age (years) } \\
\hline $25-54$ & 1.00 & $0.74(0.58-0.93)$ & $0.61(0.49-0.77)$ & $0.66(0.52-0.85)$ & $<0.001$ \\
\hline $55-74$ & 1.00 & $0.79(0.62-0.99)$ & $0.73(0.58-0.92)$ & $0.73(0.55-0.96)$ & 0.06 \\
\hline \multicolumn{6}{|l|}{ Sex } \\
\hline Male & 1.00 & $0.78(0.61-0.99)$ & $0.66(0.52-0.83)$ & $0.69(0.54-0.89)$ & 0.004 \\
\hline Female & 1.00 & $0.77(0.62-0.97)$ & $0.69(0.55-0.87)$ & $0.72(0.55-0.95)$ & 0.02 \\
\hline \multicolumn{6}{|l|}{ CVD mortality } \\
\hline \multicolumn{6}{|l|}{ Age (years) } \\
\hline $25-54$ & 1.00 & $0.81(0.60-1.10)$ & $0.63(0.46-0.85)$ & $0.64(0.46-0.90)$ & 0.01 \\
\hline $55-74$ & 1.00 & $0.75(0.56-1.00)$ & $0.75(0.56-0.99)$ & $0.77(0.55-1.09)$ & 0.2 \\
\hline \multicolumn{6}{|l|}{ Sex } \\
\hline Male & 1.00 & $0.82(0.60-1.13)$ & $0.74(0.55-1.00)$ & $0.74(0.53-1.02)$ & $>0.2$ \\
\hline Female & 1.00 & $0.76(0.57-1.00)$ & $0.66(0.49-0.87)$ & $0.69(0.49-0.98)$ & 0.03 \\
\hline \multicolumn{6}{|l|}{ CHD mortality } \\
\hline \multicolumn{6}{|l|}{ Age (years) } \\
\hline $25-54$ & 1.00 & $0.87(0.60-1.26)$ & $0.64(0.44-0.93)$ & $0.62(0.41-0.94)$ & 0.04 \\
\hline $55-74$ & 1.00 & $0.71(0.50-1.01)$ & $0.74(0.52-1.05)$ & $0.61(0.40-0.95)$ & 0.15 \\
\hline \multicolumn{6}{|l|}{ Sex } \\
\hline Male & 1.00 & $0.83(0.57-1.21)$ & $0.67(0.46-0.96)$ & $0.66(0.44-0.98)$ & 0.11 \\
\hline Female & 1.00 & $0.75(0.53-1.07)$ & $0.74(0.52-1.06)$ & $0.61(0.39-0.97)$ & 0.19 \\
\hline
\end{tabular}


same group. Some other, mostly case-control, studies found a positive association [16, 17]. Very recent data from the Iowa Women's Health Study cohort [29] revealed that consumption of 1-3 cups of coffee per day could be protective of total and CVD death. Another study of two large cohorts [30] revealed no adverse association between coffee consumption and risk of developing CHD. The effects of coffee and caffeine on the certain risk factors for CVD, such as blood pressure and serum cholesterol, have also been reported. A long-term prospective study found that coffee drinking is associated with a small increase in blood pressure [31] and another study found that modest doses of caffeine may increase blood pressure [32]. However, a study by Winkelmayer et al. [33] discovered that consumption of coffee in women is not associated with an increasing risk of developing hypertension. Coffee has been reported to raise lipid parameters. However, there is clear evidence that filtered coffee is free of two cholesterolraising ingredients (cafestol and kahweol) [34]; moreover a positive effect of chronic intake of caffeine on serum lipids has been reported [35]. We have previously found a graded inverse association between coffee consumption and type 2 diabetes [7], and in this large cohort study we have now established a reduced risk of total, CVD and CHD mortality among patients who drank 2 cups or more of coffee daily. This association was relatively strong and not affected by the other confounding or effect-modifying factors included in the analyses. After adjustment for the other CVD potential risk factors, the result even became somewhat stronger.

It has been suggested that oxidative stress is the common pathogenic factor leading to insulin resistance, beta cell dysfunction, impaired glucose tolerance and finally to type 2 diabetes [36]. Furthermore, this mechanism has been implicated as the underlying cause of both the macrovascular and microvascular complications associated with type 2 diabetes [37-40]. Blood glucose fluctuation during postprandial glycaemic excursions in people with impaired glucose tolerance or type 2 diabetes may contribute even more significantly to oxidative stress than chronic elevation of blood glucose [41]. There is much evidence from experimental studies that the formation of free radicals and presence of oxidative stress are a direct consequence of hyperglycaemia [42-44], while postprandial hyperglycaemia is a significant predictor of CVD both in non-diabetic subjects and diabetic patients $[25,45,46]$. So preventing hyperglycaemia means protecting cells from further progression of diabetes and its complications. Previous studies from diverse countries have revealed that higher coffee consumption was consistently associated with a lower prevalence of hyperglycaemia, and, in particular, coffee consumption seems to lower postprandial hyperglycaemia $[47,48]$. In our most recent study before this one, we also found lower fasting insulin values and lower risk of hyperinsulinaemia among coffee consumers with long-term exposure [49], which may be interpreted to mean that coffee consumption possibly improves insulin sensitivity, thereby preventing hyperglycaemia and oxidative stress. It has also been suggested that coffee may prevent hyperglycaemia by interfering in different stages of glucose metabolism and glycaemic regulation [49]. For instance, inhibition or retardation of the action of $\alpha$-glucosidase by chlorogenic acid is one of the most important mechanisms [50]. The inhibition of this enzyme is an effective approach to control of postprandial hyperglycaemia.

There are several strengths and limitations in our study. First, we had a homogenous study population with a large number of diabetic patients, randomly selected from the population. Second, the mean follow-up of 20.8 years was sufficiently long and the CVD events were ascertained without losses of follow-up. In addition, patients with CHD and stroke at baseline survey, and patients with type 1 diabetes at baseline and during follow-up were excluded from the analysis. Lack of data on the duration and the severity of diabetes, glucose control, and type of treatment used for management of diabetes are major limitations of our study. Reliance on self-report for data on coffee drinking may also constitute a limitation, although we believe that Finns accurately report their coffee drinking habits. In any case, any possible misclassification of the exposure is unlikely to be systematically related to the outcome. We also cannot completely exclude either the possibility and effects of residual confounding due to measurement error in the assessment of confounding factors, or the potential role of some unmeasured factors, such as dietary factors.

In conclusion, our study revealed a consistent inverse relationship between coffee consumption and the risk of total, CVD, and CHD mortality among patients with type 2 diabetes. These data will complement the gradually growing body of data that provides more evidence for the overall beneficial effects of coffee consumption in relation to type 2 diabetes. The most probable mechanisms involved are the antioxidant activity of coffee's components and their effect on the different stages of glucose metabolism, leading to improved control of hyperglycaemia. However, investigations in other populations are required in order to confirm our findings. Studies designed to better knowledge of coffee's components and their effects on diabetes and CVD complications should be greatly encouraged.

Acknowledgements This study was supported by grants from Juho Vainio Foundation, the Finnish Foundation for Cardiovascular Research and the Academy of Finland (grants 46558, 204274, and 205657).

Duality of interest The authors are not aware of any duality of interest. 


\section{References}

1. Fuller JH, Shipley MJ, Rose G, Jarrett RJ, Keen H (1983) Mortality from coronary heart disease and stroke in relation to degree of glycemia: the Whitehall study. Br Med J 287:867870

2. Nathan DM (1993) Long-term complications of diabetes mellitus. N Engl J Med 328:1676-1685

3. Stamler J, Vaccaro O, Neaton J, Wentworth D for the Multiple Risk Factor Intervention Trial Research Group (1993) Diabetes, other risk factors, and 12-year cardiovascular mortality for men screened in the multiple risk factor intervention trial. Diabetes Care 16:434-444

4. Abel ED (2005) Myocardial insulin resistance and cardiac complications of diabetes. Curr Drug Targets Immune Endocr Metabol Disord 5:219-226

5. Van Dam RM, Feskens EJ (2002) Coffee consumption and risk of type 2 diabetes mellitus. Lancet 360:1477-1478

6. Salazar-Martinez E, Willett WC, Ascherio A et al (2004) Coffee consumption and risk of Type 2 diabetes in men and women. Ann Intern Med 140:1-8

7. Tuomilehto J, Hu Gang, Bidel S, LindstrÎm J, Jousilahti P (2004) Coffee consumption and risk of type 2 diabetes mellitus among middle-aged Finnish men and women. JAMA 291:1213-1219

8. Van Dam RM, Hu FB (2005) Coffee consumption and risk of type 2 diabetes: a systematic review. JAMA 294:97-104

9. Myers MG, Basinski A (1992) Coffee and coronary heart disease. Arch Intern Med 152:1767-1772

10. Kawachi I, Colditz GA, Stone CB (1994) Does coffee drinking increase the risk of coronary heart disease? Results from a metaanalysis. Br Heart J 72:269-275

11. Greenland S (1993) A meta-analysis of coffee, myocardial infarction, and coronary death. Epidemiology 4:366-374

12. Willett WC, Stampfer MJ, Manson JE et al (1996) Coffee consumption and coronary heart disease in women. A ten-year follow-up. JAMA 275:458-462

13. Woodward M, Tunstall-Pedoe H (1999) Coffee and tea consumption in the Scottish heart health study follow up: conflicting relations with coronary risk factors, coronary disease, and all cause mortality. J Epidemiol Community Health 53:481-487

14. Kleemola P, Jousilahti P, Pietinen P, Vartiainen E, Tuomilehto J (2000) Coffee consumption and the risk of coronary heart disease and death. Arch Intern Med 160:3393-3400

15. Panagiotakos DB, Pitsavos C, Chrysohoou C, Kokkinos P, Toutouzas P, Stefanadis C (2003) The J-shaped effect of coffee consumption on the risk of developing acute coronary syndromes: the CARDIO2000 case-control study. J Nutr 133:3228-3232

16. Happonen P, Voutilainen S, Salonen JT (2004) Coffee drinking is dose-dependently related to the risk of acute coronary events in middle-aged men. J Nutr 134:2381-2386

17. Cornelis MC, El-Sohemy A, Kabagambe EK, Campos H (2006) Coffee, CYP1A2 genotype, and risk of myocardial infarction. JAMA 295:1135-1141

18. Vartiainen E, Jousilahti P, Alfthan G, Sundvall J, Pietinen P, Puska P (2000) Cardiovascular risk factor changes in Finland, 1972-1997. Int J Epidemiol 29:49-56

19. WHO MONICA Project Principal Investigators (1988) The World Health Organization MONICA Project (monitoring trends and determinants in cardiovascular disease): a major international collaboration. J Clin Epidemiol 41:105-114

20. World Health Organization Study Group on Diabetes Mellitus (1985) Diabetes Mellitus: Report of a WHO Study Group. WHO Technical Report Series No 727

21. World Health Organization Consultation (1999) Definition, diagnosis and classification of diabetes mellitus and its compli- cations. Part 1: diagnosis and classification of diabetes mellitus. WHO, Geneva

22. Hu G, Eriksson J, Barengo NC et al (2004) Occupational, commuting, and leisure-time physical activity in relation to total and cardiovascular mortality among Finnish subjects with type 2 diabetes. Circulation 110:666-673

23. Hu G, Jousilahti P, Barengo NC, Qiao Q, Lakka TA, Tuomilehto J (2005) Physical activity, cardiovascular risk factors, and mortality among Finnish adults with diabetes. Diabetes Care 28:799-805

24. Cox DR (1972) Regression models and life tables (with discussion). JR Stat Soc B 34:187-220

25. DECODE Study Group (2001) Glucose tolerance and cardiovascular mortality: comparison of fasting and 2-hour diagnostic criteria. Arch Intern Med 161:397-405

26. Hu G, Jousilahti P, Qiao Q, Katoh S, Tuomilehto J (2005) Sex differences in cardiovascular and total mortality among diabetic and non-diabetic individuals with or without history of myocardial infarction. Diabetologia 48:856-861

27. Wei M, Gaskill SP, Haffner SM, Stern MP (1998) Effects of diabetes and level of glycemia on all-cause and cardiovascular mortality. The San Antonio Heart Study. Diabetes Care 21:11671172

28. Lehto S, Ronnemaa T, Pyorala K, Laakso M (1996) Predictors of stroke in middle-aged patients with non-insulin-dependent diabetes. Stroke 27:63-68

29. Andersen LF, Jacobs DR Jr, Carlsen MH, Blomhoff R (2006) Consumption of coffee is associated with reduced risk of death attributed to inflammatory and cardiovascular diseases in the Iowa Women's Health Study. Am J Clin Nutr 83:1039-1046

30. Lopez-Garcia E, van Dam RM, Willett WC et al (2006) Coffee consumption and coronary heart disease in men and women: a prospective cohort study. Circulation 113:2045-2053

31. Klag MJ, Wang NY, Meoni LA et al (2002) Coffee intake and risk of hypertension: the Johns Hopkins precursors study. Arch Intern Med 162:657-662

32. Hartley TR, Lovallo WR, Whitsett TL (2004) Cardiovascular effects of caffeine in men and women. Am J Cardiol 93:10221026

33. Winkelmayer WC, Stampfer MJ, Willett WC, Curhan GC (2005) Habitual caffeine intake and the risk of hypertension in women. JAMA 294:2330-2335

34. Weusten-Van der Wouw MP, Katan MB, Viani R et al (1994) Identity of the cholesterol-raising factor from boiled coffee and its effects on liver function enzymes. J Lipid Res 35:721-733

35. Du Y, Melchert HU, Knopf H, Braemer-Hauth M, Gerding B, Pabel E (2005) Association of serum caffeine concentrations with blood lipids in caffeine-drug users and nonusers - results of German National Health Surveys from 1984 to 1999. Eur J Epidemiol 20:311-316

36. Ceriello A, Motz E (2004) Is oxidative stress the pathogenic mechanism underlying insulin resistance, diabetes, and cardiovascular disease? The common soil hypothesis revisited. Arterioscler Thromb Vasc Biol 24:816-823

37. Brownlee M (2001) Biochemistry and molecular cell biology of diabetic complications. Nature 414:813-820

38. Tesfamariam B (1994) Free radicals in diabetic endothelial cell dysfunction. Free Radic Biol Med 16:383-391

39. Pieper GM, Gross GJ (1998) Oxygen free radicals abolish endothelium dependent relaxation in diabetic art aorta. Am J Physiol 255:H825-H833

40. Rösen P, Nawroth PP, King G, Moller W, Tritschler HJ, Packer L (2001) The role of oxidative stress in the onset and progression of diabetes and its complications: a summary of a Congress Series sponsored by UNESCO-MCBN, the American Diabetes Association and the German Diabetes Society. Diabetes Metab Res Rev $17: 189-212$ 
41. Hirsh IB, Brownlee M (2005) Should minimal blood glucose variability become the gold standard of glycemic control? J Diabetes Complicat 19:178-181

42. Graier WF, Simecek S, Kukovetz WR, Kostner GM (1996) High glucose-induced changes in endothelial $\mathrm{Ca}^{2+} / \mathrm{EDRF}$ signaling are due to generation of superoxide anions. Diabetes 45:13861395

43. Diedrich D, Skopec J, Diedrich A, Dai FX (1994) Endothelial dysfunction in mesenteric resistance arteries of diabetic rats: role of free radicals. Am J Physiol 266:H1153-H1161

44. Tesfamariam B, Brown ML, Deykin D, Cohen RA (1990) Elevated glucose promotes generation of endothelium-dependent vasoconstrictive prostanoids in rabbit aorta. J Clin Invest 85:929932

45. Qiao Q, Pyörälä K, Pyörälä M et al (2002) Two-hour glucose is a better risk predictor for incident coronary heart disease and cardiovascular mortality than fasting glucose. Eur Heart J 23:1267-1275
46. The DECODE Study Group (2003) Gender difference in all-cause and cardiovascular mortality related to hyperglycaemia and newly diagnosed diabetes. Diabetologia 46:608-617

47. Van Dam RM, Dekker JM, Nijpels G, Stehouwer CDA, Bouter LM, Heine RJ (2004) Coffee consumption and incidence of impaired fasting glucose, impaired glucose, and type 2 diabetes: the Hoorn Study. Diabetologia 47:2152-2159

48. Yamaji T, Mizoue T, Tabata S et al (2004) Coffee consumption and glucose tolerance status in middle-aged Japanese men. Diabetologia 47:2145-2151

49. Bidel S, Hu G, Sundvall J, Kaprio J, Tuomilehto J (2006) Effects of coffee consumption on glucose tolerance and serum glucose and insulin levels - a cross sectional analysis. Horm Metab Res $38: 38-43$

50. Matsui T, Ueda T, Oki T, Sugita K, Terahara N, Matsumoto K (2001) Alpha-glucosidase inhibitory action of natural acylated anthocyanins. 2. Alpha-glucosidase inhibition by isolated acylated anthocyanins. J Agric Food Chem 49:1952-1956 